\title{
SEGURANÇA DO PACIENTE NA PERCEPÇÃO DE ACADÊMICOS DE ENFERMAGEM*
}

\author{
Patrícia Ilha ${ }^{1}$, Vera Radünz ${ }^{2}$, Francis Solange Vieira Tourinho ${ }^{2}$, Monique Mendes Marinho ${ }^{1}$
}

RESUMO: O estudo buscou conhecer a compreensão de acadêmicos em enfermagem a respeito da segurança do paciente a partir de seu contato com a prática. Estudo exploratório e descritivo, de natureza qualitativa, realizado em 2014, com 103 alunos do curso de graduação em Enfermagem de uma universidade federal do Sul do Brasil. Os alunos relataram como principais ações para a segurança do paciente, os cuidados com medicações e a integridade do paciente. Em relação à prática, consideraram o cuidado com o paciente e o comportamento individual e coletivo. Também citaram os erros de outros profissionais e a empatia com o paciente. Demonstram conhecimento sobre a cultura de segurança do paciente, mas esse ainda está relacionado ao empirismo, precisando de maior aprofundamento teórico-científico. Além disso, consideraram a segurança como algo pontual, e não processual e onipresente.

DESCRITORES: Segurança do Paciente; Enfermagem; Educação em Enfermagem; Cultura; Estudantes de Enfermagem.

\section{PATIENT SAFETY FROM THE PERSPECTIVE OF NURSING STUDENTS}

\begin{abstract}
The study sought to determine the understanding of nursing students in relation to patient safety based on their practical nursing experience. This was an exploratory, descriptive, qualitative study conducted in 2014, with 103 undergraduate nursing students from a federal university in southern Brazil. The students reported that the main patient safety actions entailed precautions with medications and patient integrity. With regard to practices, they referred to precautions with patients and their individual and collective behaviors. They also mentioned errors committed by other professionals and the quality of empathy toward patients. They demonstrated knowledge of the patient safety culture, but more on an empirical level, with a need to delve deeper on a theoretical and scientific level. They also view safety as something ad hoc, not as procedural or pervasive.
\end{abstract}

DESCRIPTORS: Patient Safety; Nursing; Nursing Education; Culture; Nursing Students.

\section{SEGURIDAD DEL PACIENTE EN LA PERCEPCIÓN DE ESTUDIANTES DE ENFERMERÍA}

RESUMEN: El estudio buscó conocer la comprensión de estudiantes de enfermería respecto de la seguridad del paciente a partir de su contacto con la práctica. Estudio exploratorio y descriptivo, de naturaleza cualitativa, realizado en 2014 con 103 alumnos del curso de grado en Enfermería de una universidad federal del Sur de Brasil. Los alumnos mencionaron como principales acciones de seguridad del paciente el cuidado con medicaciones y la integridad del paciente. Respecto de la práctica, consideran el cuidado con el paciente y el comportamiento individual y colectivo. También citaron los errores de otros profesionales y la empatía con el paciente. Demuestran conocimiento sobre la cultura de seguridad del paciente, pero este aún está relacionado al empirismo, necesitando de mayor profundización teórica y científica. Además, consideran a la seguridad como aspecto puntual, y no procesal y omnipresente.

DESCRIPTORES: Seguridad del Paciente; Enfermería; Educación en Enfermería; Cultura; Estudiantes de Enfermería.

*Artigo extraído da dissertação intitulada: "A cultura de segurança do paciente na ótica dos acadêmicos de enfermagem". Universidade Federal de Santa Catarina, 2014.

${ }^{1}$ Enfermeira. Doutoranda em Enfermagem. Universidade Federal de Santa Catarina. Florianópolis, SC, Brasil.

${ }^{2}$ Enfermeira. Doutora em Enfermagem. Docente de Enfermagem da Universidade Federal de Santa Catarina. Florianópolis, SC, Brasil.

Autor Correspondente:

Recebido: 22/10/2015

Patrícia Ilha

Finalizado: 13/07/2016

Universidade Federal de Santa Catarina

R. Europa, 228 - 88036-135 - Florianópolis, SC, Brasil

E-mail: ilha.patricia@gmail.com 


\section{INTRODUÇÃO}

As reflexões a respeito da segurança do paciente surgem por volta de 300 a.C. com Hipócrates, o pai da medicina, que já trazia a prática segura como conceito básico para as práticas médicas, com o aforismo "primum non nocere"; ou seja, em primeiro lugar, não causar dano ${ }^{(1)}$. No decorrer dos séculos, dúvidas e incertezas maiores foram aparecendo, exigindo ações mais específicas e pontuais para o cuidado seguro e que acompanhasse o desenvolvimento tecnológico.

Proporcionar segurança ao paciente significa reduzir, a um mínimo aceitável, o risco de danos desnecessários nos cuidados de saúde, considerando a somatória dos recursos humanos e materiais e o contexto em que a ação foi tomada, diante do risco de não tratamento ou de outro tratamento. Tratase, então, de reduzir atos inseguros nos processos assistenciais e usar as melhores práticas descritas, de forma a alcançar os melhores resultados para o paciente ${ }^{(2)}$.

Diversas estratégias foram elaboradas pelos órgãos responsáveis pelo controle da segurança do paciente, com a finalidade de desenvolver nos profissionais a prestação de cuidados seguros, através de pesquisas que pautassem essas ações, de forma a comprovar que um dos principais fatores que colaborava para isso era o processo de formação dos profissionais ${ }^{(3)}$.

Durante sua formação, os acadêmicos entram em contato direto com a assistência e estão expostos a diversos fatores que podem levar ao evento adverso, tais como fatores organizacionais; déficit de informações; e pessoais, como estresse psicológico e físico e sobrecarga de atividades, principalmente relacionados à falta do cuidado a si mesmo, que acabam sendo levados para a vida profissional depois de formados ${ }^{(2,4-5)}$.

Assim, é imprescindível que todos esses fatores sejam trabalhados durante a formação acadêmica, pois eles darão suporte ao desenvolvimento da cultura de segurança do paciente específica ao profissional que está sendo formado ${ }^{(2,6)}$. Para isso, deve-se conhecer a compreensão dos acadêmicos acerca da segurança do paciente, de forma a identificar as dificuldades, para que sejam superadas, e as potencialidades, para que sejam desenvolvidas e aplicadas para qualificar o serviço prestado e solidificar esse processo de implantação da segurança do paciente de forma natural, para que não apenas o apliquem, mas também o repliquem quando se tornarem profissionais.

Os profissionais, ao se formarem, assumem a responsabilidade como gestores desses cuidados prestados, e seu convívio direto com os demais profissionais propicia-lhes maior articulação para desenvolverem ações voltadas à segurança do paciente. O compromisso ético estabelece o comprometimento com a saúde do ser humano e da coletividade, impondo-os a atuar na promoção, proteção e recuperação da saúde e reabilitação das pessoas. Dessa forma, preconiza-se, para a seguridade do paciente, assistência de enfermagem livre de danos decorrentes de imperícia, negligência ou imprudência ${ }^{(7)}$.

Pautados nesses princípios, com o objetivo de conhecer a compreensão dos acadêmicos em Enfermagem a respeito da segurança do paciente a partir de seu contato com a prática, buscamos responder ao seguinte questionamento: "Qual é a cultura de segurança do paciente sob a ótica de acadêmicos da graduação em Enfermagem em uma universidade federal no Sul do Brasil?"

\section{METODOLOGIA}

Trata-se de um estudo de abordagem qualitativa, do tipo exploratório descritivo, que permite identificar diversos pontos através dos relatos dos sujeitos, propiciando explorar e compreender as opiniões, entendendo que os seres humanos são únicos e atribuem significados únicos às suas experiências. É descritivo, seguindo o preceito de que foram descritas as características, os valores e as opiniões de determinada população, fenômeno ou a relação entre variáveis, não havendo grupos nem períodos comparativos correlacionados ${ }^{(8)}$.

O ambiente de aplicação do estudo foi o Curso de Graduação em Enfermagem de uma universidade federal do Sul do Brasil, criado em 24 de janeiro de 1969. Esse curso oferece 74 vagas por ano, divididas em duas turmas com ingresso em março e agosto. Nele, são desenvolvidas estratégias que favoreçam 
a formação do enfermeiro como cidadão crítico e criativo(9).

O trabalho foi aprovado pelo Certificado de Apresentação para Apreciação Ética (CAAE), n. 24625513.9.0000.0121, e pelo Comitê de Ética em Pesquisa com Seres Humanos, através do sistema de submissão da Plataforma Brasil, sob n. 526.148, de 10 de fevereiro de 2014.

A população selecionada para este estudo foi constituída por 131 alunos, dos quais 28 decidiram não responder ao questionário, restando uma amostra total de 103 alunos. Os critérios de inclusão foram: estar regularmente matriculado no Curso de Graduação em Enfermagem no semestre de 2014/1 e cursando as disciplinas centrais da $4^{a}$ a $8^{a}$ fase de ambos os currículos em vigência na época. Os critérios de exclusão foram: alunos em atividade de intercâmbio nacional ou internacional e alunos que se ausentaram ou tiveram suas matrículas trancadas por tempo superior a um mês durante o curso ou que, por algum motivo, realizaram as atividades de forma não presencial durante o semestre.

A escolha dessa população se deve ao fato de as disciplinas dessas fases apresentarem atividades práticas, nas quais o aluno já possui contato com o ambiente profissional e/ou atividades simuladas (laboratório), executando na prática seus conhecimentos e cultivando hábitos de segurança do paciente.

Como instrumento de coleta dos dados, foi elaborado um questionário composto por quatro questões que abordavam a compreensão dos alunos sobre a segurança do paciente, sendo três delas abertas: "O que você considera ações de segurança para com o paciente?"; "Como você as põe em prática no seu dia a dia, no laboratório, nos locais de estágio curricular e/ou extracurricular?"; "O que faz você refletir sobre a segurança do paciente no cotidiano de suas vivências?", e uma fechada, na qual o aluno pôde optar por qual estratégia de segurança do paciente, definida no instrumento, ele necessita aprimorar-se, podendo selecionar mais de uma alternativa ou, ainda, sugerir outra temática.

A coleta de dados foi realizada em duas etapas. A primeira deu-se na primeira semana de aula, no mês de março de 2014, durante um período cedido pelos professores, de forma que a pesquisadora ou seu auxiliar explicaram detalhadamente como preencher o instrumento e a relevância do estudo para produção de conhecimento, assim como a necessidade da autorização através da assinatura do Termo de Consentimento Livre e Esclarecido (TCLE), deixando-os livres para qualquer questionamento e para preencher o questionário. A segunda etapa foi a busca ativa aos acadêmicos que não se encontravam na aula durante a aplicação do questionário, realizada por meio de endereço eletrônico e contato pessoal, durante os meses de março e abril de 2014.

Para organizar as questões, foram transcritas manualmente em arquivos de $W_{0 r d}{ }^{\circledR}$ de acordo com a questão $(1,2$ ou 3$)$ e pela fase em que o aluno se encontrava $\left(4^{a}, 5^{a}, 6^{a}, 7^{a}\right.$ ou $8^{a}$ fase). Feito isso, os dados foram exportados para o software NVivo 10 for Windows ${ }^{\circledR}$, no qual foram analisados, pois esse é um recurso que auxilia na organização e na estruturação, promovendo uma visão mais ampla dos dados qualitativos ${ }^{(10)}$.

Para análise dos dados, foi adotada a metodologia organizacional da Análise de Conteúdo de Bardin, conjunto de técnicas que analisa a comunicação utilizando procedimentos sistemáticos e objetivos da descrição dos conteúdos das mensagens.

No primeiro momento, foi analisado o conteúdo no plano cronológico e epistemológico; no segundo, após leitura exaustiva das questões, trabalhou-se com a associação das palavras; e, no terceiro e quarto momento, foram organizadas a análise, a codificação de resultados, a categorização e as interferências das informações com auxílio do software. Foram utilizados também os três polos de organização na análise de conteúdo: pré-análise; exploração do material; e tratamento dos resultados.

\section{RESULTADOS}

Primeiramente foram identificadas as informações relativas à questão um do instrumento, categorizadas com base na leitura exaustiva dos dados e estudo dos termos e temáticas que permeiam a segurança do paciente. Esses dados foram categorizados levando-se em consideração os principais pontos abordados em cada categoria e o objetivo da questão, em que o acadêmico deveria expressar sua concepção sobre ações de segurança, a forma como poderia demonstrar e confirmar a dimensão 
da cultura de segurança do paciente que ele já tem estabelecida e/ou que está em processo de desenvolvimento, assim como as potencialidades e dificuldades de entendimento do tema (Tabela 1).

Na sequência, foi aplicada igual metodologia com a questão dois do instrumento, considerando a compreensão do seu objetivo, que era analisar a congruência entre o entendimento teórico sobre a segurança do paciente e sua potencial aplicação prática (Tabela 2).

Posteriormente, foi aplicada a metodologia também à questão três, com o objetivo de entender as fundamentações/potencialidades/pilares nos quais o acadêmico busca recursos para seu aprendizado e toma como exemplo para atitudes que levam a comportamentos (Tabela 3).

Tabela 1 - Categorização dos dados qualitativos relativos à questão 1 ( $\mathrm{O}$ que você considera ações de segurança para com o paciente?). Florianópolis, SC, Brasil, 2014

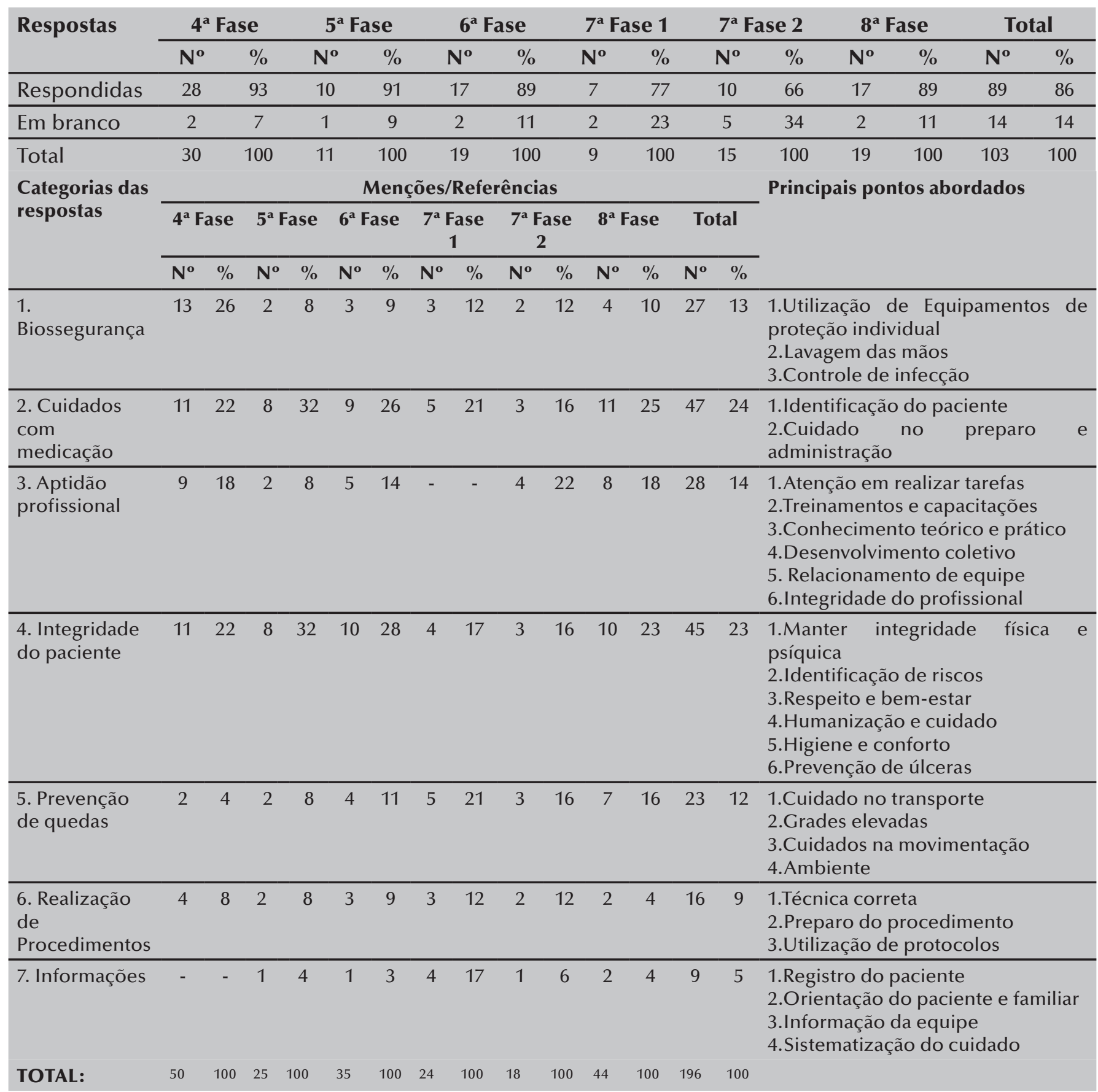


Para rever as informações da questão quatro foi realizada análise descritiva por meio de frequência e porcentagem das respostas (Tabela 4), por tratar-se de questão de múltipla escolha. Apenas na última alternativa (outro) havia espaço para informação qualitativa complementar, e não foi preenchido por nenhum participante.

Essa questão propôs que o aluno optasse pela estratégia de segurança do paciente, definida no instrumento, na qual ele necessita aprimorar-se, podendo selecionar mais de uma alternativa ou, ainda, sugerir outra temática.

Tabela 2 - Categorização dos dados qualitativos relativos à questão 2 (Como você coloca em prática as ações de segurança do paciente no seu dia a dia, no laboratório, nos locais de estágio curricular e ou extracurricular?). Florianópolis, SC, Brasil, 2014

\begin{tabular}{|c|c|c|c|c|c|c|c|c|c|c|c|c|c|c|c|c|c|c|}
\hline \multirow[t]{2}{*}{ Respostas } & \multicolumn{3}{|c|}{$4^{\mathrm{a}}$ Fase } & \multicolumn{3}{|c|}{$5^{\mathrm{a}}$ Fase } & \multicolumn{3}{|c|}{$6^{\mathrm{a}}$ Fase } & \multicolumn{2}{|c|}{$7^{\mathrm{a}}$ Fase 1} & \multicolumn{3}{|c|}{$7^{\mathrm{a}}$ Fase 2} & \multicolumn{2}{|c|}{$8^{a}$ Fase } & \multicolumn{2}{|c|}{ Total } \\
\hline & \multicolumn{2}{|c|}{$\mathrm{N}^{\mathbf{0}}$} & $\%$ & \multicolumn{2}{|c|}{$\mathbf{N}^{\mathbf{0}}$} & $\%$ & $\mathbf{N}^{\mathbf{0}}$ & $\%$ & \multicolumn{2}{|r|}{$\mathrm{N}^{\mathbf{o}}$} & $\%$ & \multicolumn{2}{|c|}{$\mathbf{N}^{\mathbf{0}}$} & $\%$ & $\mathbf{N}^{\mathbf{o}}$ & $\%$ & $\mathrm{~N}^{\mathbf{0}}$ & $\%$ \\
\hline Respondidas & \multicolumn{2}{|c|}{24} & 80 & \multicolumn{2}{|c|}{9} & 81 & 15 & 78 & \multicolumn{2}{|r|}{6} & 66 & \multicolumn{2}{|c|}{10} & 66 & 15 & 78 & 79 & 76 \\
\hline Em branco & \multicolumn{2}{|c|}{6} & 20 & \multicolumn{2}{|c|}{2} & 19 & 4 & 22 & \multicolumn{2}{|r|}{3} & 34 & \multicolumn{2}{|r|}{5} & 34 & 4 & 22 & 24 & 24 \\
\hline Total & \multicolumn{2}{|c|}{30} & 100 & \multicolumn{2}{|c|}{11} & 100 & 19 & 10 & & 9 & 100 & & 15 & 100 & 19 & 100 & 103 & 100 \\
\hline \multirow[t]{3}{*}{ Categorias } & \multicolumn{14}{|c|}{ Menções/Referências } & \multirow{3}{*}{\multicolumn{4}{|c|}{ Principais pontos abordados }} \\
\hline & \multicolumn{2}{|c|}{$4^{\mathrm{a}}$ Fase } & \multicolumn{3}{|c|}{$5^{\text {a }}$ Fase } & $\begin{array}{c}6^{a} \\
\text { Fase }\end{array}$ & \multicolumn{2}{|c|}{$7^{\mathrm{a}}$ Fase 1} & \multicolumn{2}{|c|}{$7^{a}$ Fase 2} & \multicolumn{2}{|c|}{$8^{\text {a }}$ Fase } & \multicolumn{2}{|c|}{ Total } & & & & \\
\hline & $\mathbf{N}^{\mathbf{0}}$ & $\%$ & $\mathbf{N}^{\mathbf{0}}$ & $\%$ & $\mathbf{N}^{\circ}$ & $\%$ & $\mathrm{~N}^{\mathbf{0}}$ & $\%$ & $\mathbf{N}^{\circ}$ & $\%$ & $\mathbf{N}^{\circ}$ & $\%$ & $\mathbf{N}^{\mathbf{o}}$ & $\%$ & & & & \\
\hline $\begin{array}{l}\text { 1.Aperfei- } \\
\text { çoamento } \\
\text { profissional }\end{array}$ & 5 & 14 & 4 & 33 & 1 & 4 & - & - & 4 & 25 & 1 & 5 & 15 & 13 & $\begin{array}{l}\text { 1. Atua } \\
\text { 2. Estu } \\
\text { 3. Pesc } \\
\end{array}$ & $\begin{array}{l}\text { zação } \\
\text { s } \\
\text { isas }\end{array}$ & & \\
\hline $\begin{array}{l}\text { 2.Comporta- } \\
\text { mento } \\
\text { individual e } \\
\text { coletivo }\end{array}$ & 9 & 26 & 1 & 9 & 8 & 30 & 1 & 20 & 6 & 37 & 10 & 45 & 35 & 30 & $\begin{array}{l}\text { 1. Agir } \\
\text { hones } \\
\text { respei } \\
\text { 2. Agir } \\
\text { 3. Resp }\end{array}$ & $\begin{array}{l}\text { om co } \\
\text { lade, } \\
\text { om se } \\
\text { isabil }\end{array}$ & $\begin{array}{l}\text { ência, } \\
\text { ca e } \\
\text { Irança } \\
\text { ade }\end{array}$ & \\
\hline $\begin{array}{l}\text { 3.Cuidados } \\
\text { com } \\
\text { procedimentos }\end{array}$ & 7 & 20 & - & - & 5 & 18 & 1 & 20 & 1 & 7 & 6 & 28 & 20 & 17 & $\begin{array}{l}\text { 1. Revi } \\
\text { 2. Técr } \\
\text { 3. Real } \\
\text { possív } \\
\text { 4. Cert }\end{array}$ & $\begin{array}{l}\text { r os p } \\
\text { as co } \\
\text { ar da } \\
\text { da } m\end{array}$ & $\begin{array}{l}\text { cedim } \\
\text { tas } \\
\text { elhor } f \\
\text { dicaçã }\end{array}$ & rma \\
\hline $\begin{array}{l}\text { 4.Cuidados de } \\
\text { Biossegurança }\end{array}$ & 10 & 29 & 4 & 33 & 4 & 15 & 2 & 40 & - & - & 2 & 9 & 22 & 19 & $\begin{array}{l}\text { 1. Utili } \\
\text { 2. Con } \\
\text { 3. Higi } \\
\text { 4. Utili }\end{array}$ & $\begin{array}{l}\text { ição d } \\
\text { ole de } \\
\text { ee das } \\
\text { ar pro }\end{array}$ & $\begin{array}{l}\text { EPIs } \\
\text { afecçã } \\
\text { ãos } \\
\text { colos }\end{array}$ & \\
\hline $\begin{array}{l}\text { 5.Cuidado com } \\
\text { o paciente }\end{array}$ & 4 & 11 & 3 & 25 & 9 & 33 & 1 & 20 & 5 & 31 & 3 & 13 & 25 & 21 & $\begin{array}{l}\text { 1. Paci } \\
\text { indivic } \\
\text { 2. Esta } \\
\text { e plan } \\
\text { com a } \\
\text { 3. Orie } \\
\text { 4. Sup } \\
\text { 5. Cuic }\end{array}$ & $\begin{array}{l}\text { te e s } \\
\text { alidac } \\
\text { elecer } \\
\text { amen } \\
\text { tecess } \\
\text { taçõe } \\
\text { te bio } \\
\text { dos cc }\end{array}$ & $\begin{array}{l}\text { riorida } \\
\text { de acc } \\
\text { lades } \\
\text { icossc } \\
\text { o am }\end{array}$ & $\begin{array}{l}\text { les } \\
\text { do } \\
\text { ial } \\
\text { iente }\end{array}$ \\
\hline TOTAL: & 35 & 100 & 12 & 100 & 27 & 100 & 5 & 100 & 16 & 100 & 22 & 100 & 117 & 100 & & & & \\
\hline
\end{tabular}


Tabela 3 - Categorização dos dados qualitativos relativos à questão 3 (O que faz você refletir sobre a segurança do paciente no cotidiano de suas vivências?). Florianópolis, SC, Brasil, 2014

\begin{tabular}{|c|c|c|c|c|c|c|c|c|c|c|c|c|c|c|c|c|c|c|c|}
\hline \multirow[t]{2}{*}{ Respostas } & & \multicolumn{3}{|c|}{$4^{a}$ Fase } & \multicolumn{3}{|c|}{$5^{a}$ Fase } & \multicolumn{3}{|c|}{$6^{a}$ Fase } & \multicolumn{3}{|c|}{$7^{\mathrm{a}}$ Fase 1} & \multicolumn{2}{|c|}{$7^{\mathrm{a}}$ Fase 2} & \multicolumn{4}{|c|}{$8^{\text {a }}$ Fase Total } \\
\hline & & \multicolumn{2}{|c|}{$\mathbf{N}^{\mathbf{0}}$} & $\%$ & \multicolumn{2}{|c|}{$\mathbf{N}^{\mathbf{0}}$} & $\%$ & $\mathbf{N}^{\mathbf{0}}$ & \multicolumn{2}{|c|}{$\%$} & $\mathbf{N}^{\mathbf{0}}$ & \multicolumn{2}{|l|}{$\%$} & $\mathbf{N}^{\mathbf{o}}$ & $\%$ & \multirow{2}{*}{\multicolumn{2}{|c|}{$\begin{array}{ll}\mathbf{N}^{\mathbf{0}} & \% \\
14 & 78\end{array}$}} & \multirow{2}{*}{\multicolumn{2}{|c|}{$\begin{array}{cc}\mathbf{N}^{\mathbf{0}} & \% \\
80 & 77 \\
\end{array}$}} \\
\hline Respondidas & & 2 & 5 & 83 & 9 & & 81 & 16 & & 84 & 6 & 66 & & 10 & 66 & & & & \\
\hline Em branco & & & 5 & 17 & 2 & & 19 & 3 & & 16 & 3 & 34 & 4 & 5 & 34 & 5 & 22 & 23 & 23 \\
\hline Total & & 3 & 0 & 100 & 11 & & 100 & 19 & & 100 & 9 & 100 & 0 & 15 & 100 & 19 & 100 & 103 & 100 \\
\hline \multirow[t]{3}{*}{ Categorias } & \multicolumn{14}{|c|}{ Menções/Referências } & \multirow{3}{*}{\multicolumn{5}{|c|}{ Principais pontos abordados }} \\
\hline & \multicolumn{2}{|c|}{$4^{a}$ Fase } & \multicolumn{2}{|c|}{$5^{\text {a }}$ Fase } & \multicolumn{2}{|c|}{$6^{a}$ Fase } & $\begin{array}{l}7^{a} \\
1 \\
\end{array}$ & Fase & \multicolumn{2}{|c|}{$\begin{array}{l}7^{a} \text { Fase } \\
2\end{array}$} & \multicolumn{2}{|c|}{$8^{\mathrm{a}}$ Fase } & \multicolumn{2}{|c|}{ Total } & & & & & \\
\hline & $\mathbf{N}^{\circ}$ & $\%$ & $\mathbf{N}^{\circ}$ & $\%$ & $\mathbf{N}^{\mathbf{0}}$ & $\%$ & $\mathbf{N}^{\mathbf{0}}$ & $\%$ & $\mathbf{N}^{\mathbf{0}}$ & $\%$ & $\mathbf{N}^{\mathbf{0}}$ & $\%$ & $\mathbf{N}^{\circ}$ & $\%$ & & & & & \\
\hline $\begin{array}{l}\text { 1.Empatia } \\
\text { com o } \\
\text { paciente }\end{array}$ & 9 & 34 & 3 & 34 & 1 & 6 & - & - & 3 & 30 & 3 & 21 & 19 & 24 & $\begin{array}{l}\text { 1. Co } \\
\text { pacier } \\
\text { 2. Pac } \\
\text { do cui } \\
\text { 3. Col } \\
\text { de um }\end{array}$ & $\begin{array}{l}\text { locar- } \\
\text { te } \\
\text { ente } \\
\text { dado } \\
\text { car o } \\
\text { famili }\end{array}$ & $\begin{array}{l}\text { e no } \\
\text { omo } \\
\text { pacie } \\
\text { ir }\end{array}$ & $\begin{array}{l}\text { lugar } \\
\text { depend } \\
\text { te no I }\end{array}$ & $\begin{array}{l}\text { do } \\
\text { lugar } \\
\end{array}$ \\
\hline $\begin{array}{l}\text { 2.Erros } \\
\text { de outros } \\
\text { profissionais }\end{array}$ & 10 & 38 & 6 & 66 & 10 & 55 & 2 & 66 & 4 & 40 & 5 & 36 & 37 & 46 & $\begin{array}{l}\text { 1. } \\
\text { profis } \\
\text { 2.Situ } \\
\text { expõe }\end{array}$ & $\begin{array}{l}\text { ros } \\
\text { ionais } \\
\text { çõesc } \\
\text { no pa }\end{array}$ & $\begin{array}{l}\text { realiz } \\
\text { ueosp } \\
\text { ciente }\end{array}$ & $\begin{array}{l}\text { ados } \\
\text { rofissic } \\
\text { a risco }\end{array}$ & $\begin{array}{l}\text { por } \\
\text { onais } \\
\text { S }\end{array}$ \\
\hline $\begin{array}{l}\text { 3.Aptidão do } \\
\text { profissional }\end{array}$ & 6 & 24 & - & - & 4 & 23 & - & - & 1 & 10 & 5 & 36 & 16 & 20 & $\begin{array}{l}\text { 1. Estu } \\
\text { 2. Co } \\
\text { com a } \\
\text { 3. Refl } \\
\text { 4. } \\
\text { profis } \\
\text { 5. Sob } \\
\text { 6.Próp }\end{array}$ & $\begin{array}{l}\text { los te } \\
\text { npara } \\
\text { indic } \\
\text { exão d } \\
\text { ional } \\
\text { ecarg } \\
\text { rio erı }\end{array}$ & $\begin{array}{l}\text { ricos } \\
\text { atituc } \\
\text { ações } \\
\text { as açõ } \\
\text { Respo } \\
\text { da ec } \\
\text { D. } \\
\end{array}$ & $\begin{array}{l}\text { discus } \\
\text { les prá } \\
\text { teórica } \\
\text { es } \\
\text { nsabilic } \\
\text { quipe }\end{array}$ & $\begin{array}{l}\text { sões } \\
\text { iticas } \\
\text { s } \\
\text { dade }\end{array}$ \\
\hline $\begin{array}{l}\text { 4.Próprio } \\
\text { erro }\end{array}$ & 1 & 4 & - & - & 3 & 16 & 1 & 34 & 2 & 20 & 1 & 7 & 8 & 10 & $\begin{array}{l}\text { 1. Re } \\
\text { comeI } \\
\text { 2. Risc }\end{array}$ & $\begin{array}{l}\text { lexão } \\
\text { tem. } \\
\text { os. }\end{array}$ & dos & erros & \\
\hline TOTAL: & 26 & 100 & 9 & 100 & 18 & 100 & 3 & 100 & 10 & 100 & 14 & 100 & 80 & 100 & & & & & \\
\hline
\end{tabular}

Tabela 4 - Análise descritiva dos dados relativos à questão 4 (Eu gostaria de receber treinamentos /orientações adicionais em:). Florianópolis, SC, Brasil, 2014

\begin{tabular}{lcc} 
Opção & Frequência & $\%$ \\
\hline Como praticar técnicas eficazes e seguras para melhorar a assistência ao paciente. & 61 & 59 \\
\hline Como revelar um erro a um paciente. & 62 & 60 \\
\hline Como prevenir erros de medicação. & 51 & 50 \\
\hline $\begin{array}{l}\text { Como incorporar o uso de listas de verificação (checklist) para melhorar o atendimento ao } \\
\text { paciente. }\end{array}$ & 36 & 34 \\
\hline Como avaliar um paciente com risco de eventos adversos. & 66 & 64 \\
\hline Como rever uma situação para identificar por que e como um erro ocorreu. & 49 & 47 \\
\hline Como melhorar meus registros para destacar as questões de segurança do paciente. & 51 & 49 \\
\hline Como apoiar outras pessoas que se envolveram em um erro. & 45 & 43 \\
\hline Como lidar com um erro no qual estou envolvido. & 66 & 64 \\
\hline Outro. & 1 & 1
\end{tabular}




\section{- DISCUSSÃO}

Ao analisar os resultados, percebeu-se que as falas e categorias encontradas se referem a dimensões ligadas às crenças e normas subjetivas e pouco relacionadas com conhecimentos teóricos científicos. Isso foi evidenciado quando os acadêmicos, questionados sobre questões mais teóricas, trazem fundamentações voltadas às experiências pessoais e empíricas, citando, em apenas uma categoria (relacionada à medicação), normativas e protocolos.

Para o desenvolvimento da cultura de segurança do paciente positiva, crenças, conhecimentos científicos, experiências e normas devem estar alinhados e ponderados; todavia, este estudo identificou uma dissensão desses atributos nas falas dos acadêmicos ${ }^{(11)}$.

Ao analisar as respostas da primeira questão, que buscava identificar a compreensão dos acadêmicos sobre o que consideravam ações de segurança do paciente, emergiram sete categorias, das quais a mais citada foi "cuidados com medicações" $(24 \%)$, na qual as principais falas sugeriam a identificação da medicação e os cuidados no preparo, corroborando as campanhas que vêm sendo realizadas em prol da segurança do paciente, em que se sobressai a medicação segura, dando visibilidade a essa temática através de protocolos de orientação e campanhas encetadas pelos órgãos governamentais. Uma delas é o Protocolo de Segurança na Prescrição, Uso e Administração de Medicamentos, elaborado pela Agência Nacional de Vigilância Sanitária (ANVISA) e a Fundação Oswaldo Cruz (Fiocruz), o qual almeja promover práticas seguras no uso de medicamentos em estabelecimentos de saúde, tornando-se referência nacional no desenvolvimento da temática ${ }^{(12)}$.

Dessa forma, o reconhecimento por parte dos acadêmicos vai ao encontro da literatura, que aponta o processo de formação como o principal responsável pela instituição de uma cultura de segurança positiva e desenvolvimento de estratégias para com a manipulação de medicamentos e prevenção de erros de medicação. Além da grande visibilidade que esse tipo de erro vem tendo através da mídia e por ser um dos erros mais comuns que comprometem a segurança do paciente ${ }^{(13)}$. Essa foi, portanto, a única categoria que teve referência direta a um protocolo ou referencial estabelecido.

A segunda mais citada foi a integridade do paciente (23\%). Os pontos mais abordados nas falas foram relacionados a: manter integridade física e psíquica; identificar riscos; respeito e bem-estar; humanização e cuidado; higiene e conforto; e prevenção de úlceras.

Quando analisamos essas falas, identificamos que foram contempladas ações bem pontuais, de forma que em nenhum momento os acadêmicos expressaram a abordagem articular e sistemática da segurança do paciente como algo amplo. Conclui-se que, ao mesmo tempo em que eles têm noção do que permeia a segurança do paciente, eles não conseguem vê-la como um processo que passa por diversas etapas interligadas, desde a formação dos profissionais e estruturação das instituições, até a prevenção do erro ou a redução de danos de um erro já consumado.

O processo de formação dos profissionais da saúde ainda é voltado para as competências restritas às suas disciplinas, e sem formação multiprofissional e transdisciplinar. Desse modo, cada profissão aborda a segurança do paciente dentro de seu campo de atuação e restrito às suas atividades, quando na verdade ela transpassa esses aspectos. A construção de uma cultura de segurança positiva envolve um trabalho conjunto, que necessita de uma formação voltada para tal, com currículos unificados e multidisciplinares que propiciem o pensar juntos em estratégias e soluções voltadas à segurança ${ }^{(14)}$.

A segunda questão se voltava para compreender como eles articulavam os conhecimentos teóricos sobre a segurança do paciente, com as experiências que realizavam nos estágios e atividades práticas. Surgiram cinco categorias, sendo as mais citadas: o comportamento individual e coletivo (30\%), abordando o agir com coerência e honestidade, ética e respeito; agir com segurança; e responsabilidade.

Estudos apontam que esses aspectos são identificados na práxis profissional e são citados nas mesmas perspectivas encontradas neste estudo, uma vez que os profissionais referem um déficit em sua formação, relacionado a questões de ética, responsabilidade e comportamento frente ao erro. Citam, ainda, como estratégia para superação desses fatores, a construção do plano de cuidados para segurança do paciente de forma coletiva e voltada ao cotidiano e ao contexto que encontram no seu ambiente de trabalho e à discussão da temática entre a equipe. Estratégias essas emergentes em outros 
tópicos discutidos neste estudo, corroborando com a perspectiva de que as ações coletivas estão diretamente envolvidas com o desempenho da segurança ${ }^{(15-16)}$.

Outra categoria emergente desse tópico foi o cuidado com o paciente (21\%), sendo citados o paciente e sua individualidade; estabelecer prioridades e planejamento de acordo com as necessidades; orientações; suporte biopsicossocial; e cuidados com o ambiente. Essa reflexão dirigida à integridade do paciente é importante para o desenvolvimento do perfil profissional.

Em um artigo cujos autores buscaram compreender como os enfermeiros realizam assistência humanizada diante dos aprendizados adquiridos em sua vivência acadêmica, constatou-se que a forma como eles foram instruídos e os exemplos que tiveram durante sua formação influenciaram na maneira como viam o cuidado e as condutas realizadas junto ao paciente ${ }^{(17)}$. Uma das competências descritas nas Diretrizes Curriculares Nacionais dos Cursos de Graduação em Enfermagem apresenta, entre seus objetivos, "a garantia da capacitação dos profissionais de saúde em relação à autonomia e ao discernimento, a fim de assegurar a integralidade da atenção e a humanização do atendimento dos indivíduos, das famílias e da comunidade"(18).

A terceira questão indagava o que os fazia refletir sobre a segurança do paciente. Os participantes verificaram que o erro dos outros $(46 \%)$ os faziam pensar na segurança do paciente ou em situações que os profissionais expuseram o paciente a riscos. Em seus relatos, eles deram a entender que a análise do erro não pode ser individual, mas sistêmica, e que cada vez mais a assistência à saúde é desenvolvida em ambientes dinâmicos e especializados onde ocorrem interações complexas entre fisiopatologia, equipe, infraestrutura, processos, normas e procedimentos. Desta forma, a prevenção da ocorrência de erros não está relacionada exclusivamente à mudança de conduta dos profissionais, o que vai ao encontro de outros estudos que trazem também uma reflexão madura e atualizada dos acadêmicos acerca dos erros, fugindo do modelo punitivista ${ }^{(17)}$.

Outro ponto presente nessa questão foi a empatia (24\%). As respostas a abordaram de forma mais empírica nas falas e, às vezes, encaradas pelo viés da caridade, situação essa comum também entre os profissionais, devido à dificuldade de relacionarem os conceitos do cuidado humanizado com o conhecimento científico ${ }^{(19)}$.

A partir do momento que a profissão se desenvolveu como disciplina, há a necessidade de agregar esse conhecimento aos seus valores e práticas, de modo que as teorias de enfermagem auxiliam nesse embasamento. Uma delas é a Teoria do Cuidado Humano, de Margaret Jean Watson, que define o conceito da empatia no cuidado contemporâneo como a capacidade de compreender os sentimentos de outra pessoa e comunicá-la de tal experiência, baseada nos pilares cognitivos, afetivos e comportamentais, que facilitam a formação de um vínculo terapêutico, o qual é fundamental na realização de um cuidado qualificado, tendo implicâncias relevantes à segurança do paciente não apenas como responsabilidade técnica, mas também de maneira humanizada ${ }^{(19)}$.

Quando questionados sobre o que desejavam aprimorar dentro da temática da segurança do paciente, a maioria dos acadêmicos assinalou questões que envolviam o erro, avaliação de risco e técnicas para melhorar a segurança, ratificando outros estudos, como um realizado na Califórnia, em 2013, que buscou compreender a percepção dos alunos de medicina sobre a cultura de segurança do paciente. Nesse estudo, os alunos revelaram o desejo de receber uma formação complementar a respeito de segurança do paciente para melhorar a sua experiência educacional, pois se sentem inseguros e indecisos sobre a ação a ser tomada, principalmente quando se deparam com o erro ou com o risco de este ocorrer ${ }^{(17,10)}$. Isso reflete a necessidade do aprimoramento científico, demonstrando mais uma vez que essa lacuna ainda é um desafio para o estabelecimento da cultura de segurança.

Se bem analisado, porém, esse é um dos pontos mais palpáveis de transformação em potencialidade, visto que é a mudança das crenças e culturas que já acompanham os acadêmicos desde sua formação como ser social, e essa mudança é mais complexa do que o processo de conhecimento e aprendizado que pode ser construído na parceria instituição-sujeito e sociedade. Há que se considerar também que o acesso à informação permite que o sujeito reavalie seus valores culturais, reestruturando suas crenças e proporcionando uma mudança de hábitos e atitudes, de forma que a informação, discussão e expressão constituem o início de uma mudança construtiva, estruturada e positiva ${ }^{(11,20)}$. 
Antes de executarmos qualquer ação ou pensarmos em estratégias para o desenvolvimento da cultura de segurança do paciente, é importante compreendermos o entendimento que as pessoas envolvidas têm sobre a temática e quais são os fatores que a permeiam, principalmente quando partimos do princípio de que diversas questões culturais, não apenas de cunho individual, mas também de responsabilidade coletiva, estão envolvidas, sendo necessário reconhecer as potencialidades e fragilidades dentro desse processo.

Por meio deste estudo, pudemos identificar que os acadêmicos têm um conhecimento sobre a cultura de segurança, relacionado ao empirismo de suas vivências, não só na academia, mas também às suas experiências no decorrer da vida. Portanto, ainda carecem de uma sustentação teórica e de uma conexão racional das ações a serem realizadas fundamentais para a execução de um cuidado seguro.

A academia facilita o desenvolvimento dessas habilidades, uma vez que é um local que oportuniza um espaço adequado para a discussão das ações e possui estrutura para trabalhar os aspectos de formação de um profissional crítico.

Há necessidade, portanto, da formulação de novas metodologias que propiciem uma estratégia efetiva para o desenvolvimento da educação para segurança do paciente no processo de formação do enfermeiro, considerando as crenças já trazidas por eles e fortalecendo o desenvolvimento teórico científico para uma visão ampliada, articulada e fundamentada da segurança do paciente.

Como limitação do estudo, apontamos o instrumento que apresentava questões predeterminadas, as quais podem ter limitado a abrangência das respostas. Como sugestão, seria interessante a realização de uma entrevista aberta, oportunizando a expressão e aprofundamento do tema e seus elementos por parte dos acadêmicos e/ou, ainda, a observação de sua prática no campo de estágio para verificar se condizem com os discursos.

\section{REFERÊNCIAS}

1. Zambon LS. Medicina Net. [Internet]. Riscos e eventos adversos:uma realidade alarmante.. Porto Alegre: MedicinaNet. [atualizado em 04 mar 2009; acesso em 02 jun 2014]. Disponível:

http://assinantes.medicinanet.com.br/conteudos/biblioteca/901/introducao__primum_non_nocere.htm

2. World Health Organization (WHO). The conceptual framework for the international classification for patient safety. Technical Report and Technical Annexes. 2009. [acesso em 16 set 2016]. Disponível:

http://www.who.int/patientsafety/taxonomy/icps_full_report.pdf

3. Gasperi P. O cuidar de si como uma dimensão da cultura de segurança do paciente [tese]. Florianópolis (SC): Universidade Federal de Santa Catarina; 2013.

4. Santos VEP, Radünz V. Estresse de acadêmicas x segurança do paciente. Rev. enferm. UERJ. 2011; 19(4): 616-20.

5. Radünz V, Carraro TE. Cuidar de si para cuidar do outro. In: Reibnitz K. Horr L, Souza ML, Spricigo JS, organizadoras. O processo de cuidar, ensinar e aprender o fenômeno das drogas: políticas de saúde, educação e Enfermagem. Módulo 2- especialização no fenômeno das drogas área de redução da demanda. Florianópolis: Departamento de Enfermagem/UFSC, 2003, p. 99-112.

6. Corral-Mulato S, Baldissera VDA, dos Santos JL, Philbert LAS, Bueno SMV. Estresse na vida do acadêmico em enfermagem: (des)conhecimento e prevenção. Invest. educ. enferm 2011; 29(1): 109-17.

7. Conselho Federal de Enfermagem (COFEN). Resolução n ${ }^{\circ} 311$ de 08 de fevereiro de 2007. Aprova a Reformulação do código de ética dos profissionais de enfermagem. Rio de Janeiro: COFEN; [Internet] 2007. [acesso em 04 jun 2014]. Disponível: http://se.corens.portalcofen.gov.br/codigo-de-etica-resolucao-cofen-3112007

8. da Silva EL, Menezes EM. Metodologia de pesquisa e elaboração de dissertação. $3^{\mathrm{a}}$ ed. Florianópolis: Laboratório de Ensino a Distância da UFSC; 2001. 
9. Universidade Federal de Santa Catarina (UFSC). Guia dos estudantes do curso de graduação em enfermagem / Universidade Federal de Santa Catarina; coordenadoras Sayonara de Fátima Faria Barbosa, Lucia Nazareth Amante, Astrid EggertBoehs. Florianópolis; 2008.

10. Lage MC. Utilização do software NVivo e m pesquisa qualitativa: uma experiência em EaD. Educ. Tem. Dig. 2011; 12(1): 198-226.

11. Moutinho K, Roazzi A. As teorias da ação racional e da ação planejada: relações entre intenções e comportamentos. Aval psicol. 2010; 2(9): 279-87.

12. Ministério da Saúde (BR). Portaria $N^{\circ} 529$, de $1^{\circ}$ de abril de 2013. Institui o Programa Nacional de Segurança do Paciente (PNSP). [Internet] 1 abr 2013. [acesso em 15 jun 2014]. Disponível:

http://bvsms.saude.gov.br/bvs/saudelegis/gm/2013/prt0529_01_04_2013.html

13. de Souza ECC, Bueno AAB, Fassarella CS. Segurança do paciente no ambiente hospitalar: os avanços na prevenção de eventos adversos no sistema de medicação. Revista Rede de Cuidados em Saúde. 2013; 7(1): 1-8.

14. Bohomol E, Freitas MAO, Cunha ICKO. Enseñanza sobre seguridaddel paciente enelpregradoensalud: reflexiones sobre saberes y prácticas. Interface (Botucatu). [Internet] 2016; 20(58) [acesso em 6 mar 2016 ]. Disponível: http://dx.doi.org/10.1590/1807-57622015.0699

15. Weiss FP, Stein DB, Cassola T, Gehlen MH, Rosa RT, Ilha S. Segurança do paciente na práxis do cuidado de enfermagem: percepção de enfermeiros. Ciencia y Enfermería. 2015; 21(3): 37-47.

16. Schwonke CRGB, Filho Lunardi WD, Lunardi GL, da Silveira RS, Rodrigues MCS, Guerreiro MO. Cultura de segurança: a percepção dos profissionais de enfermagem intensivistas. Enferm Global. 2016; 41(1): 220-31.

17. Bogarin DF, Zanetti ACB, Brito MFP, Machado JP, Gabriel CS, Bernardes A. A segurança do paciente: conhecimento de alunos de graduação em enfermagem. Cogitare enferm. 2014; 19(3): 491-7.

18. Lazzari DD, Jacobs LG, Jung W. Humanização da assistência na enfermagem a partir da formação acadêmica. Rev Enferm UFSM. 2012; 1(2): 116-24.

19. Savieto RM, Leao ER. Asistencia en Enfermería y Jean Watson: una reflexión de empatía. Esc. Anna Nery. 2016; 20(1): 198-202.

20. Bowman C, Neeman N, Sehgal N. Enculturation of unsafe attitudes and behaviors: student perceptions of safety culture. Acad Med. 2013; 88(6): 802-10. 